\title{
Adjustment of Pointing \& Tracking System of ERT5 and Results of Some Bright Source Observations
}

\author{
Ismail Yusifov \\ Erciyes University, Dept. Astronomy and Space Sciences, \\ 38039, Kayseri, TURKEY \\ E-mail: yusifoveerciyes.edu.tr \\ National Academy of Sciences of Azerbaijan, Institute of Physics, \\ Baku 370143, AZERBAIJAN \\ E-mail: ismail.yusifov@gmail.com
}

\section{Ibrahim Küçük}

Erciyes University, Dept. Astronomy and Space Sciences,

38039, Kayseri, TURKEY

E-mail: kucuk@erciyes.edu.tr

In this report we inform on adjustment of pointing and tracking system and calibration procedures of ERT5. Also we inform on the test of $1420 \mathrm{MHz}$ HI receiver SpectraCyber ( SC ) from Radio Astronomy Supplies [1] which is installed on ERT5 and the results of some bright source observations with it. Errors of pointing of ERT5 before correction were more than $7^{\circ}$. After the first stage of correction these errors had been reduced to nearly $2^{\circ}$ level. Testing of the Neutral hydrogen ( $\mathrm{HI}$ ) receiver SC shows that the system is functioning properly. We also discuss the first observations made in Turkey at the $1420 \mathrm{MHz}$ line and continuum and other results of some observations of Galactic and extragalactic bright sources.

VII Microquasar Workshop: Microquasars and Beyond

Foca, Izmir, Turkey

September $1^{\text {st }}-5^{\text {th }} 2008$ 


\section{Introduction}

5m diameter Erciyes University Educational Radio Telescope ( ERT5 ) is the first working radio telescope in Turkey. The telescope was constructed by the financial support of Erciyes University Research Development Projects Department (Project No: FB. 03.12 and FB. 03. 18) and a $5 \mathrm{~m}$ satellite antenna which was presented by Turkish TELECOM.

Converting the satellite antenna to the radio telescope consists of constructing a rotation system with step motors and encoders for the precise pointing of the telescope toward the celestial bodies, developing PC controlled electronics, receiver system and software for the general control of the telescope.

ERT5 has two systems of receivers: a wide band radiometer with central frequencies of $v_{\mathrm{o}}$ $=10.5 \mathrm{GHz}$ and bandwidth $\Delta v=1 \mathrm{GHz}$ and Neutral hydrogen receiver SC from Radio Astronomy Supplies ( RAS ). Information on the technical details of ERT5 and radiometer calibration procedures can be found in [2] and [3]. These studies show that all the systems of the ERT5 in general function satisfactorily. However there are big errors in the pointing system.

In this report we will shortly discuss pointing error measurements, their corrections and the test of ERT5 $1420 \mathrm{MHz} \mathrm{HI}$ receiver.

\section{ERT5 Pointing Error Measurements and Their Correction}

Pointing errors of ERT5 have been measured with the help of a mounted optical guide, by pointing telescope to the Sun, Moon, and various bright stars in the sky. During June 2008 session we carried out nearly several hundred error measurements. Only part of them were useful for error function estimation and pointing corrections of ERT5. The result of pointing correction is shown in Figure1. Improvements are evident but for precise pointing further measurements and additional corrections are required.

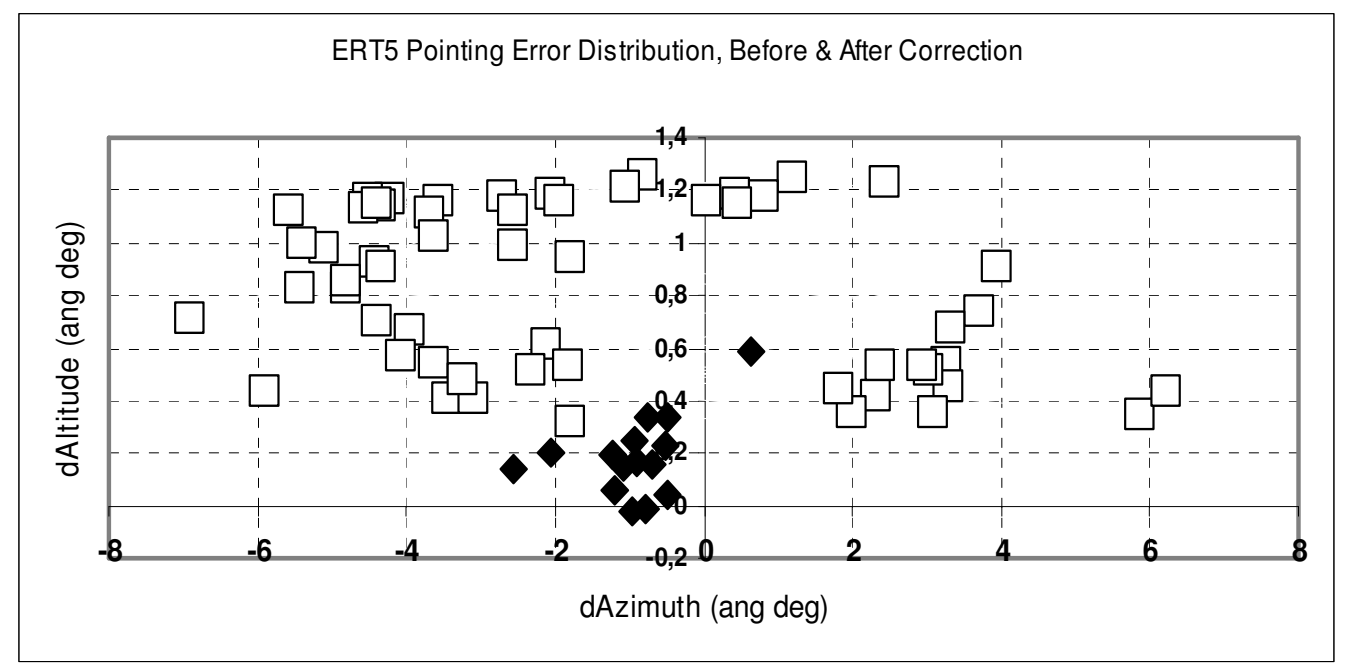

Figure 1: Pointing error scatter distribution for June 2008 status of ERT5. Empty squares correspond before and filled ones correspond after pointing and tracking correction of ERT5. 
Theoretical value of the beam width of ERT5 at the frequency of $1420 \mathrm{MHz}$ is nearly $2^{\circ}, 5$. From Figure 1 it is seen that, after correction, pointing error is also around this value. In this case, we can attempt to test our HI receiver, pointing telescope in various directions in the Galaxy. Results of these test observations are described in the next sections.

\section{First Observation of $1420 \mathrm{MHz}$ HI line in Turkey}

As it is noted, ERT5 has two receivers: A $10.5 \mathrm{GHz}$ radiometer and a $\mathrm{HI}$ receiver from the RAS. Test of the radiometer was carried out last year, on the base of the Sun and Moon observations and results were described in [3]. The improvement of pointing and tracking allows us to test also neutral hydrogen receiver of ERT5. Observations have been carried out in several directions of the galactic plane and one result is shown in Figure 2. Our data haven't been corrected for the Local Standard of Rest. This may be one of the reasons for $30 \mathrm{~km} / \mathrm{s}$ deviation of maximum of line profile from $0 \mathrm{~km} / \mathrm{s}$. The HI line in other directions of Galactic longitudes has similar appearance.

HI Observation of the Galactic Plane at $\mathrm{I}^{\mathrm{i}}=70^{\circ}$ with SpectraCyber-1 on ERT5

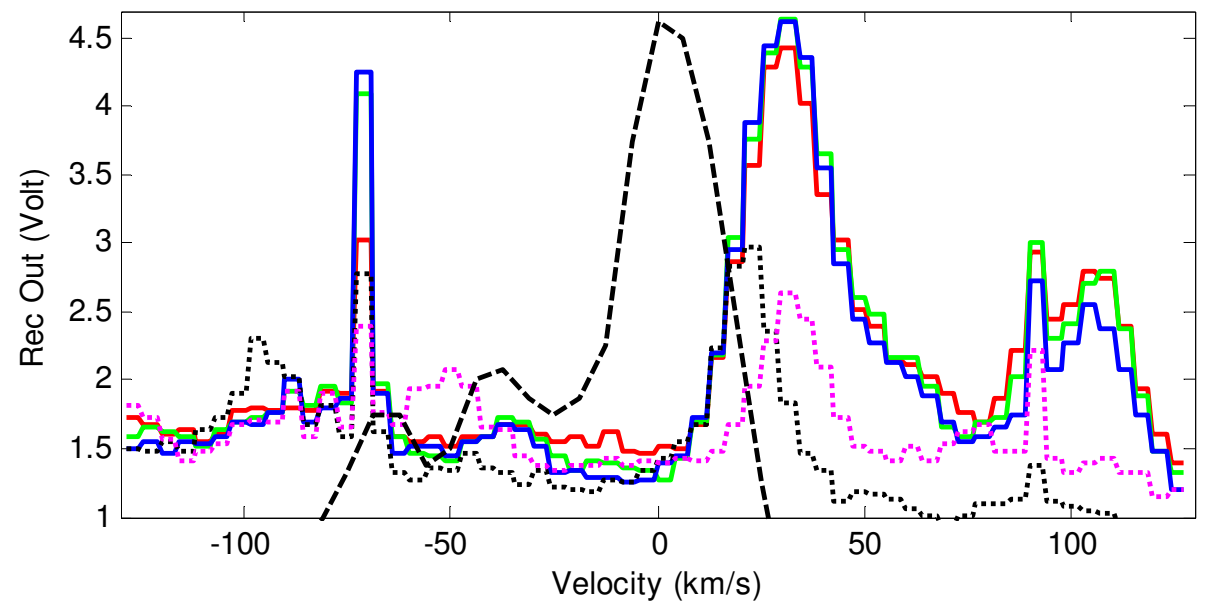

Figure 2: The first Observation of the $1420 \mathrm{MHz}$ HI line in Turkey. 2 dotted lines were measured nearly $5^{\circ}-6^{\circ}$ from the Galactic plane and 3 solid lines within $\pm 1^{\circ}$ around the Galactic plane. The dashed line from [4], corresponds near $1^{11}=80^{\circ}$ Galactic longitude. Very sharp peaks near -70 and $80 \mathrm{~km} / \mathrm{s}$ are probably digital noise from the computer.

\section{Observation of Cygnus A by ERT5 and Estimation of Beam Shape}

One of brightest radio sources in the sky is radio galaxy Cygnus A. According to early observations angular width of Cygnus A is nearly 2'. As the theoretical beam width of the ERT5 at $1420 \mathrm{MHz}$ is nearly 150', observation of Cygnus A may give us some information about it.

A scan of Cygnus A by ERT5 and fitted background of observational data are shown in Figure 3(Left). The data after subtracting the background are also shown in the same figure. Gauss fitted shape of these residuals may be accepted as ERT5 beam shape at the first 
approximation. For the half power width of this Gauss distribution we obtain 135'. Consequently, to a first approximation as the value of the HPBW of the ERT5 we may accept nearly 134', which correspond to the directivity of nearly 35dB. From the observation of the nearest mountain, the sky and the Moon we have made preliminary estimation of the following main parameters of ERT5: main beam efficiency $\eta_{B}=0.9$; aperture efficiency $\eta_{A}=0.58$; antenna loses $\eta_{t}=0.98$ and receiver noise temperature $T_{r}=274{ }^{\circ} \mathrm{K}$.
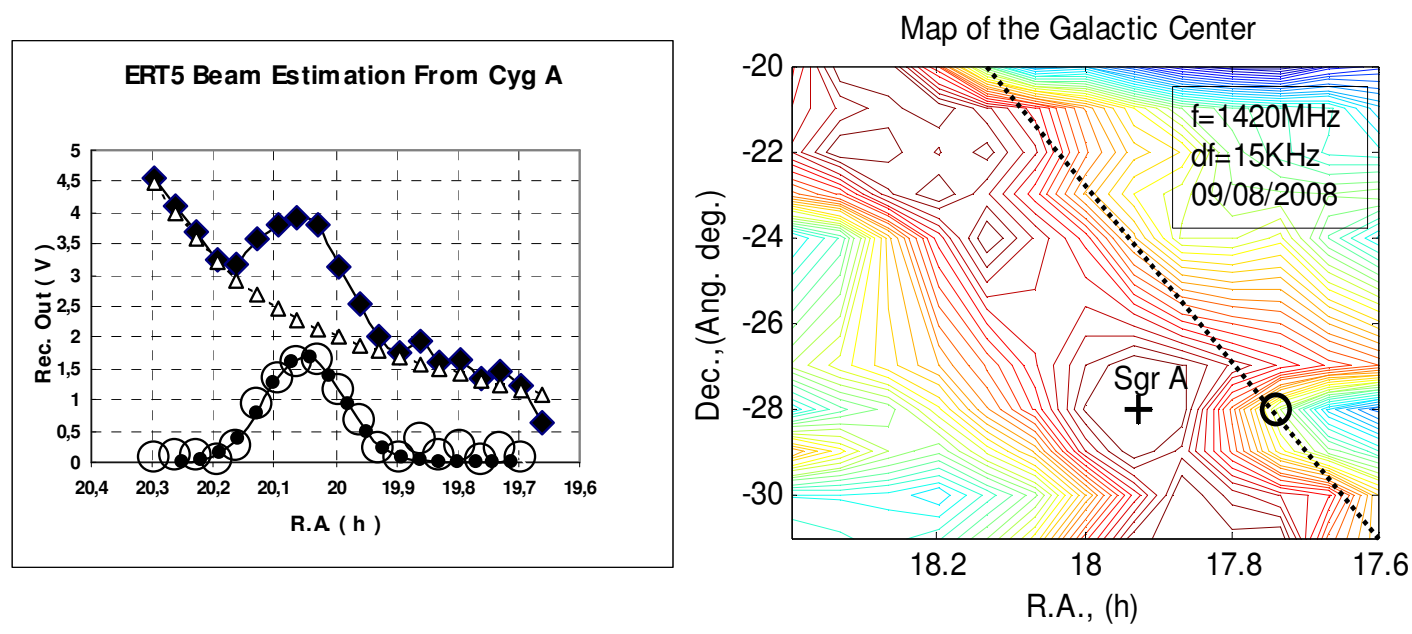

Figure 3: Left: Observation of Cygnus A with ERT5. Measured outputs of the receiver shown by big filled squares. Fitted baseline of observations is shown with empty triangles and dotted line. The observed values after subtracting the baseline are shown with empty circulars. Gauss fitting of these data are shown with dots. Right: Map of the Galactic central region obtained at the $1420 \mathrm{MHz}$ continuum. Selectable band width of the receiver was $15 \mathrm{KHz}$. Expected posision of the Galactic plane is shown by dotted line.

\section{Mapping of the Galactic Central Region in Continuum at $1420 \mathrm{MHz}$}

The next step of test procedures of ERT5 system is mapping or scanning various Galactic or extragalactic sources in continuum. One of interesting radio sources of all time is the Galactic central region containing galactic black hole SgrA. In order to map this region we have scanned $12^{\circ} \times 12^{\circ}$ central region of the Galaxy with one degree steps. The center of this region is located at $18^{\mathrm{h}}$ right ascensions $-26^{\circ}$ declinations. A map of the Galactic central region obtained after reduction of these observational data is shown in Figure 3 (Right). As noted in section 2, at present time the pointing error of the ERT5 is nearly $2^{\circ}$. Just for this reason the calculated and observed positions of SgrA and the Galactic plane do not coincide with each other.

\section{Observation of Supernova Remnant by ERT5}

In order to test tracking possibilities of ERT5 more precisely, we also tried to point the telescope to other bright radio sources and scan them. Supernova Remnant (SNR) Cas A is the second brightest radio sours after the Sun. In order to detect Cas A we scanned a $2^{\circ} \times 14^{\circ}$ rectangular region located at $1^{11}=111^{\circ} 47^{\prime}$ and $b^{11}=-02^{\circ} 07^{\prime}$ in Galactic longitude and latitude 
coordinates. In this experiment, Cas A has been clearly seen at the edge of the region which is near $1=111^{\circ}$ and is shown in Figure 4 Left. Low level of signal is probably due to a pointing error.

Another region, where we have pointed ERT5 is W51 complex which is located at $\alpha=19^{\mathrm{h}}$ $23^{\mathrm{m}}$ and $\delta=14^{\circ} 30^{\prime}$. This is a very complex region, consisting of SNR, HII region, and molecular clouds. Again, due to pointing error, maximum signal is observed not from the expected, but the scan near declination $\delta=15^{\circ} 30^{\prime}$ and is shown in Figure 4 Right. Signal level here is high and the complex structure of the source is clearly seen.
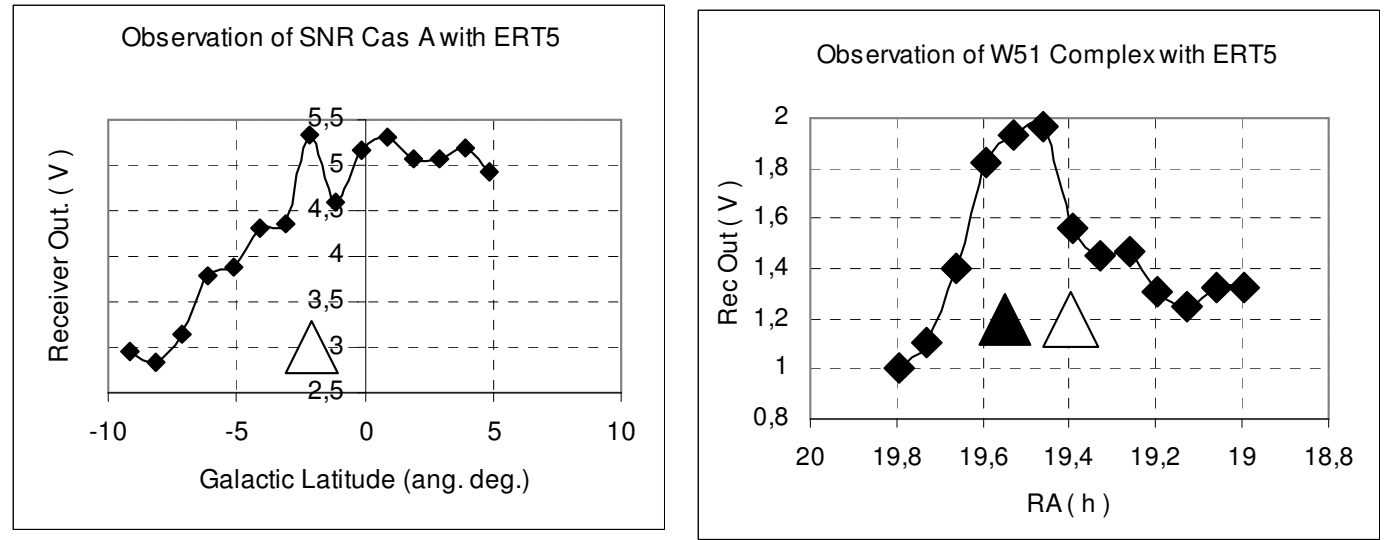

Figure 4: Left: Observation of SNR Cas A with ERT5. Expected position of Cas A on Galactic latitude is shown by triangle. Low level of signal is most probably connected with $2^{\circ}$ pointing error in azimuth direction. Right: ERT5 observation of W51 complex. Expected and observed positions of W51 are shown by empty and filled triangles, correspondently.

\section{Conclusions}

Test observations show that at present time pointing and tracking error of Erciyes University radio telescope ERT5 is order of $2^{\circ}$. Both receivers $(10.5 \mathrm{GHz}$ radiometer and HI receiver SpectraCyber (SC) from RAS ) function satisfactorily. After improving pointing precision, ERT5 with SC can be successfully used in educational process of practical radio astronomy and for galactic and extragalactic HI observations

\section{References}

[1] RAS, http://www.radioastronomysupplies.com/

[2] I. Yusifov, I. Küçük, etal., "Technical Properties of ERT-5”, XV National Astronomy Conference, Istanbul Culture University, Istanbul, Turkey, pp. 939-946, 28 August - 1 Sep. 2006.

[3] I. Yusifov, I. Küçük, etal., "Calibrating Observations of ERT-5 Radio Telescope”, International Conference of TFD-24, İnönü Universities, Malatya, Turkey, 28 - 31 August, 2007.

[4] J.D. Kraus, Radio Astronomy, McGraw Hill, 1982 